\title{
Customized orthogonalization via deflation algorithm with applications in face recognition
}

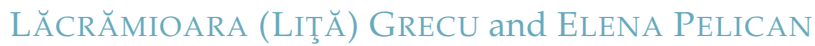

\section{ABSTRACT.}

The face recognition problem is a topical issue in computer vision. In this paper we propose a customized version of the orthogonalization via deflation algorithm to tackle this problem. We test the new proposed algorithm on two datasets: the well-known ORL dataset and an own face dataset, CTOVF; also, we compare our results (in terms of rate recognition and average quiery time) with the outcome of a standard algorithm in this class (dimension reduction methods using numerical linear algebra tools).

Acknowledgements. The authors are grateful to Achiya Dax for his invaluable advice and comments, on an early version of this manuscript.

\section{REFERENCES}

[1] Chen, J. and Saad, Y., Lanczos Vectors versus Singular Vectors for Effective Dimension Reduction, IEEE Transactions on Knowledge and Data Engineering, 21 (2009), No. 8, 1091-1103

[2] Dax, A., Orthogonalization Via Deflation: A Minimum Norm Approach for Low-Rank Approximations of a Matrix, J. Comput. Appl. Math., 234 (2010), No. 11, 3091-3103

[3] Dax, A., A minimum norm approach for low-rank approximations of a matrix, SIAM J. Matrix Anal. Appl., 30 (2008), No. 1, 236-260

[4] Delac, K., Grgic, M. and Grgic, S., Independent Comparative Study of PCA, ICA, and LDA on the FERET Data Set, International Journal of Imaging Systems and Technology, 15 (2006), No. 5, 252-260

[5] Delac, K., Grgic, M. and Liatsis, P., Appearance-based Statistical Methods for Face Recognition, Proceedings of the 47th International Symposium ELMAR-2005 focused on Multimedia Systems and Applications, Zadar, Croatia, 2005, 151-158

[6] Duda, R. O., Hart, P. E. and Stork, D. G., Pattern Classification, second ed., Wiley-Interscience, New York, 2001

[7] Elden, L., Matrix Methods in Data Mining and Pattern Recognition, SIAM, Philadelphia, 2007

[8] Glineur, F., Lu, L., Van Dooren, P. and Wang, X., Extended Lanczos Bidiagonalization for Dimension Reduction in Information Retrieval, 8th International Conference on Natural Computation (ICNC'12), Maui, Hawaii, 2012

[9] Hotelling, H., Analysis of a complex of statistical variables into principal components, J. Educ. Psychol., 24 (1933), 417-441, 498-520

[10] Jolliffe, I. T., Principal Component Analysis, Springer, Second Edition, 2002

[11] Kirby, M. and Sirovich, L., Application of the Karhunen-Loeve Procedure for the Characterization of Human Faces, IEEE Transactions on Pattern Analysis and Machine Intelligence, 12 (1990), No. 1, 103-108

[12] Liţă, L. and Pelican, E., A Low-Rank Tensor-Based Algorithm for Face Recognition, submitted

[13] Martinez, A. M., and Kak, A. C., PCA versus LDA, IEEE Transactions on Pattern Analysis and Machine Intelligence, 23 (2001), No. 2. 228-233

[14] Narasimha Murty M., and Susheela, Devi V., Pattern Recognition. An Algorithmic Approach, Springer, First Edition, 2011

[15] Pearson, K., On lines and planes of closest fit to systems of points in space, Phil. Mag., 2 (1901), No. 6, 559-572

[16] Pelican, E., and Grecu, L., Comparison Between Some Matrix Methods with Applications in Pattern Recognition, Applied Linear Algebra Conference, May 24-28, 2010, Novi Sad

Received: 28.01.2013; In revised form: 16.10.2013; Accepted: 15.01.2014

2010 Mathematics Subject Classification. 68T10, 15A18.

Key words and phrases. Pattern recognition, dimension reduction, eigenvalue, eigenvector, singular values.

Corresponding author: Elena Pelican; epelican@univ-ovidius.ro 
[17] Pelican, E. and Liţă (Grecu), L., Solving the Pattern Recognition Problem with some Low-Rank Approximation Based Algorithms, XIème Colloque Franco-Roumain de Mathématiques Appliquées, 24-30 August, 2012, Bucarest

[18] Pelican, E. and Grecu, L., Low-Rank Matrix Methods in Pattern Recognition, Balkan Conference on Operational Research (BALCOR) (2009), ISBN: 973-86979-9-9

[19] Pentland, A., Starner, T., Etcoff, N., Masoiu, A., Oliyide, O. and Turk, M., Experiments with Eigenfaces, Looking at People Workshop, IJCAI'93, 1993

[20] Sirovich, L. and Kirby, M., Low-dimensional Procedure for the Characterization of Human Faces, Journal of the Optical Society of America A - Optics, Image Science and Vision, 4 (1987), No. 3, 519-524

[21] Turk, M. and Pentland, A., Eigenfaces for Recognition, Journal of Cognitive Neuroscience, 1 (1991), 71-86

[22] Turk, M. and Pentland, A., Face Recognition using Eigenfaces, Computer Vision and Pattern Recognition Proceedings (1991), 586-591

Department of Mathematics And COMPUter SCience

OVIDIUS UNIVERSITY CONSTANTA

BLVD MAMAia, 124, CONSTANTA, ROMANIA

E-mail address: Igrecu, epelican@univ-ovidius.ro 